\title{
Illyrian Personal Anthroponyms
}

\author{
Giancarlo T. Tomezzoli ${ }^{1}$, Rainhardt S. Stein ${ }^{2}$ \\ ${ }^{1}$ Etno-Archaeological Observatory, Munich, Germany \\ ${ }^{2}$ Universiteit Utrecht, Uithof, Holland \\ Email: gt21949@gmx.de,rstein@uu.nl
}

How to cite this paper: Tomezzoli, G. T. \& Stein, R. S. (2021). Illyrian Personal Anthroponyms. Archaeological Discovery, 9, $1-15$. https://doi.org/10.4236/ad.2021.91001

Received: October 19, 2020

Accepted: November 16, 2020

Published: November 19, 2020

Copyright $\odot 2021$ by author(s) and Scientific Research Publishing Inc. This work is licensed under the Creative Commons Attribution International License (CC BY 4.0).

http://creativecommons.org/licenses/by/4.0/ (c) (i) Open Access

\begin{abstract}
This article follows a previous publication dedicated to Venetic personal anthroponyms. In the present article, we investigate Illyrian personal anthroponyms from the Corpus Inscriptionum Latinarum, a continuously updated Latin inscription catalogue covering the Roman Empire period and its provinces. The Illyrian anthroponyms appear deeply latinised and graecised and the meaning of their roots was identified by comparing each one of them with corresponding lexemes in the present surviving Slavic languages. The result is that the Illyrian personal anthroponyms having Slavic roots is $45.93 \%$, which permits to estimate that during the Roman Empire period, i.e. about five cen., roughly $46 \%$ of the Illyrian population had Slavic ascendancies. This highlights that Slavs were already present in areas incorporated by the Roman Empire well before the VII cen. A.D. the date, according to the generally accepted theory, of the Slav late arrival in Europe. The logical consequence is that this theory is wrong and should be rejected.
\end{abstract}

\section{Keywords}

Illyrians, Anthroponyms, Veneti, Slavs, Late Arrival, Roman Empire, C.I.L., Linguistics

\section{Introduction}

A great number of publications deal with the origins, the languages and the territories of the ancient Illyrians (I.). Because they did not left written documents, the conclusions of both classical and contemporary scholars concerning I. matters are normally divergent (Wilkes, 1992). In essence, it is possible to say that the entity constituted by the ancient I. is rather uncertain. The approach to acquire information about the I. through I. anthroponyms was object of some publications. One of them (Krahe, 1929) listed I. anthroponyms systematically according to their appearance in the Corpus Inscriptionum Latinarum (C.I.L.) 
without providing their etymology. Purpose of this article is to deepen the knowledge about the I. by considering the etymology of the I. personal anthroponyms. This article follows a similar one dedicated to the etymology of Venetic (V.) personal anthroponyms (Stein \& Tomezzoli, 2020) and inter alia provides a reply to the question set out in a recent publication (Pigozzo, 2020): "the I. belonged to the paleo Slavic family and, consequently, were they related to the ancient V.?"

\section{Illyrian Personal Anthroponyms}

The above mentioned publication (Krahe, 1929), on pages 1-131, lists I. personal anthroponyms. We considered them one by one and only those having Slavic roots were retained in alphabetic order in Table 1.

Each record in Table 1 comprises: Re, the progressive record number, I. Anth, the I. anthroponyms sharing the same Slavic root, R. Mean, the root meaning, Sl. Lexem., the corresponding lexemes in present surviving Slavic languages from which the root meaning was inferred, C.I.L., the corresponding C.I.L. entry if available, $\mathrm{p}$, the page in said publication where each I. anthroponym resides and V.T1.n, the I. anthroponyms correspondence, if any, with V. anthroponyms in Table 1 in the corresponding publication (Stein \& Tomezzoli, 2020). Table 1 should be read bearing in mind the conventions of Table 2 .

Table 1. Illyrian anthroponym list.

\begin{tabular}{|c|c|c|c|c|c|c|}
\hline Re. & I. Anth. & R. Mean. & Sl. Lexem. & C.I.L. & Page & V.T1.n \\
\hline
\end{tabular}

A

1 †Adgeleius: hell - Bos. had, Blg. ад, Cro. had, O.Ch.Sl. адъ, Rus. ад, Ser. хад, Ukr. ад; C.I.L. III, 4844, p 3; V.T1.1.

$2 \nmid$ †mbisavus: granary - Bel. амбар, Bos. ambar, Cro. ambar, Rus. амбар, Ser. амбар, Ukr. амбар; C.I.L. III 13406, p 4.

$3 †$ †trans: quick - Bos. hitar, Cro. hitar, Lit. atrs, O.Blg. њдръ, Rus. хитрый, Ser. хитар, Slo. hitro; C.I.L. III, 5117, p 12; V.T1.3.

$4 \dagger$ At(t)o, Attu: shout to dogs - Bel. aту, Rus. ату, Ukr. aту; C.I.L. III 6010, 26, p 12; p 12.

$5{ }^{\star}$ Avidiaccus, Avittius, ${ }^{\star}$ Avitus: reality - Bos. java, Cro. java, Rus. явb, Ser. jaвa; C.I.L. IX, 346, p 13; C.I.L. IX 423, p 13; C.I.L. III, 3853, p 14; V.T1.5.

B

$6{ }^{\star}$ Baezocrusu, ${ }^{\star}$ Baezus: without - Bel. без, Bos. bez, Blg. без, Blg.dial. бизая, Cro. bez, Cze. bez, Lit. be, Мac. без, Pol. bez, Rus. без, Ser. без, Slo. brez, Slv. bez, Ukr. без; C.I.L. III, 14321 ${ }^{1}$, p 14; C.I.L. III, 1270, p 14; V.T1.6.

$7 \quad{ }^{*}$ Bateia, ${ }^{*}$ Bá $\varepsilon \varepsilon ı$, Bato, Batoniana, Batonius, ${ }^{*}$ Battarios, Batun: dad - Bel. баця, Rus. батя, Ukr. батя; C.I.L. III, 5031, p 16; p 16; C.I.L. III, 12779, p 17; C.I.L. III 14633, p. 20; p 20; p 20; C.I.L. III 9845, p 20; V.T1.7.

$8 \quad{ }^{*}$ Bedarus: trouble - Bel. бяда, Bul. беда, Lit. bèda, Pol. bieda, Rus. беда, Ukr. біда; C.I.L. III 917, p 20

$9 \dagger$ †ellus, ${ }^{\star}$ Bilisa: white - Bel. бел, Bos. bela, Blg. бял, Cro. bijela, Cze. bílý, Lit. balta, Mac. бело, O.Ch.Sl. бълъ, Pol. biały, Rus. белый, Ser. беo, Slo. bela, Slv. biely, Ukr. білі; p 20; C.I.L. III 6010, p 21; V.T1.8. 


\section{Continued}

10 *Beres, †Bersasius: birch - Bel. бяроза, Bos. breza, Bul. бреза, Cro. breze, Cze. bříza, Lit. beržas, Мас. бреза, O.Ch.Sl. бръза, Pol. brzoza, Rus. берёза, Ser. бреза, Slo. breza, Slv. breza, Ukr. береза; C.I.L. III 8734, p 20; C.I.L. V 3516, p 21; V.T1.9.

$11{ }^{\star}$ Bisena, ${ }^{\star B}$ Bisius, Bı $а \tau \iota$, Bizo: spring - Bel. вясна, Lit. versmé, O.Ch.Sl. весна, Rus. весна, Ukr. весна; C.I.L. V 1363, p 22; C.I.L. III 1266, p 22; p 22; C.I.L. III 2782, p 22; V.T1.10.

12 Blazziza: whim - Bel. блажь, Rus. блажь, Ukr. блажь; C.I.L. III 8292, p 23.

$13{ }^{\star}$ Boicus, ${ }^{\star}$ Boius: fighter - Bel. баец, Bos. borac, Blg. боец, Cro. borac, Cze. bojovník, Mac. борец, O.Ch.Sl воинъ, Pol. bojowiec, Rus. боец, Ser. војник, Slo. borec, Slv. vojak, Ukr. боєць; C.I.L. V 433, p 23; C.I.L. V 579, p 23; V.T1.11.

14 Boria: pine - Bos. bor, Bul. бор, Cro. bor, Cze. borovice, Mac. бор, O.Ch. Sl. боль, Rus. бор, Ser. бop, Slo. bor, Slv. borovica; C.I.L. V 7, p 24.

15 Breucus, Bricena, *Bricussa: shore - Bel. бераг, Blg. бряг, Cze. břeh, Mac. брегот, O.Ch.Sl. брьгъ, Pol. brzeg, Rus. берег, Slv. breh, Ukr. берег; C.I.L. VIII 21041, p 24; C.I.L. III 917, p 24; p 24; V.T1.13.

$16{ }^{\star}$ Brisia, ${ }^{\star}$ Brizidia, ${ }^{\star}$ Brizinus: go away! - Bel. брысь, Rus. брысь, Ukr. брись; C.I.L. V 4912, p 25; C.I.L. III 8302, p 25; C.I.L. IX 6192, p 25.

17 Bui(i)o: rowdy - Bel. буян, O.Ch.Sl. буий, Rus. буян; C.I.L. III 3790 p 25; V.T1.16.

18 *Burnia, Burnio: storm - Blg. буря, Cze. bouřka, Mac. бура, O.Ch.Sl. вътръ, Pol. burza, Rus. буря, Slv. búrka, Ukr. буря; C.I.L. X 7144, p 26; C.I.L. VIII 21041, p 26; V.T1.17.

19 Busa, Busia, Busidius, Busio, Buzetius, Buzos: alcoolic drink - Bel. буза, Rus. буза, Ukr. буза; p 26; C.I.L. IX 689, p 26; C.I.L. IX 335, p 26; C.I.L. III 10362, p 26; C.I.L. III 9929a, p 27; p 27.

C

20 Calas, †Calsasia: mud - Bos. kal, Blg. кал, Cro. kal, Mac. кал, Ser. кал, Slo. kal, Ukr. кал; p 27; C.I.L. V 2414, p 27; V.T1.18.

21 Cammica, ${ }^{\star}$ Cammius, $†$ Coemoius, $†$ Coimo, ${ }^{\star}$ Cummia: stone - Bel. камень, Bos. kamena, Blg. камък, Cro. kamen, Cze. kámen, Lit. akmuo, Мас. камен, O.Ch.Sl. камы, Pol. kamień, Rus. камень, Ser. камен, Slo. kamen, Slv. kameň, Ukr. камінь; C.I.L. V 2327, p 27; C.I.L. V 961, p 27; C.I.L. III 3792, p 31; C.I.L. III 10854, p 31; C.I.L. III 4496, p 33; V.T1.19.

22 †Candalio: shackles - Bel. кайданы, Pol. kajdany, Rus. кандалы, Ukr. кайдани; C.I.L. III $9813 \mathrm{a}, \mathrm{p} 27$.

$23{ }^{\star}$ Carpia, ${ }^{\star}$ Carp(i)us: carp - Bel. карп, Bos. karaš, Cro. karaš, Cze. kapr, Lit. karpis, Mac. крап, Pol. karp, Rus. карп, Ser. караш, Slo. krap, Slv. kapor, Ukr. короп; C.I.L. III 9839, p 28; C.I.L. III C VIII, p 28.

$24{ }^{\star}$ Catandio, ${ }^{\star}$ Cato, †Cattu, [Caturus]: hut - Bel. хата, Cze. chata, Pol. chata, Rus. хата, Slv. chata, Ukr. хата; C.I.L. III 2425, p 29; C.I.L. III 4392, p 29; C.I.L. III 5042, p 29; C.I.L. III 2779, p 29.

25 Ciasicianus, Ciassicus: hour - Bel. час, Bul. час, Mac. час, O.Ch.Sl. часъ, Rus. час; C.I.L. III 2757, p 30; C.I.L. III 9810, p 30.

26 *Clevas, Clevatus: barn - Bel. хлеў, Rus. хлев, Ukr. хлів; p 31; p 31.

27 *Cornuinus: root - Bel. корань, Bos. korijen, Blg. корен, Cro. korijen, Cze. kořen, Mac. корен, O.Ch.Sl. корєнь, Pol. korzeń, Rus. корень, Ser. корен, Slo. koren, Slv. korUkr. корінь; C.I.L. V 417, p 32; V.T1.20.

$28{ }^{\star}$ Corragos: mountain - Bel. гара, Cze. hora, Lit. krūva, O.Ch.Sl. гора, Pol. góra, Rus. гора, Slo. gora, Slv. hora, Ukr. ropa; p 32.

29 Crapus: dot - Bel. крап, Pol. kropka, Rus. крап, Ukr. крап; C.I.L. III 2979, р 32.

30 Cynna, †Cynnis: rank - Bel. чын, Bos. čin, Cro. čin, O.Ch.Sl. чинъ, Rus. чин, Ser. чин, Slo. čin, Ukr. чин; p 33; p 33. 


\section{Continued}

D

31 Dardana, ${ }^{\star}$ Dardas, ${ }^{\star}$ Darmo: gift - Bel. дap, Cro. dar, Cze. dar, Pol. dar, Rus. дap, Ser., Slo. darilo, Slv. darček, Ukr. дар; p 34; p 34; C.I.L. III 2779, p 34.

32 Dasant-, Dasantilla, Dases, Dasianius, Dasiatius, Dasimianus, Dasimius, Dasumius, Dasimos, Dasmenus, Dasmus, Das(s)ianus, Das(s)ius, Dastidius, Dasto, Dasumius, Dasummius, Daszos: to give - Bel. даваць, Bos. dati, Blg. дадеш, Cro. dati, Cze. dát, Lit. duoti, Mac. дава, O.Ch.Sl. дати, Pol. dawać, Rus. даст, Ser. дати, Slo. dajati, Slv. dat, Ukr. Давати; C.I.L. III $14316^{5}$, p 34; C.I.L. III 14774, p 35; C.I.L. III, 4276, p 35; C.I.L. VI 16774, p 35; C.I.L. X 8059140, p 35; C.I.L. IX 338, p 35; C.I.L. IX 373, p 35; C.I.L. IX 373, p 35; p 36; C.I.L. III D LXI, p 36; C.I.L. IX 390, p 36; C.I.L. III 3540, p 37; C.I.L. III 1938, p 37; C.I.L. IX 2115, p 38; C.I.L. III 8551, p 38; p 38; p 38; p 38; V. T1.26.

33 Daziscus: to oppress - Bos. tlačiti, Cro. tlačiti, Rus. давить, Ser. тлачити, Slo. tlačiti, Ukr. давити; р 40; V.T1.25.

34 Daza, Dazas, Dazanus; Dazen, Dazeta, Dazier, Dazima, Dazimos, Dazios, Dazipos, Daziscus Dazomenus, Dazos, Dazun-, Dazupos, Dazymos, ${ }^{\star}$ Dizo: the tenth - Bel. дзясяты, Bos. deseti, Blg. десетата, Cro. deseti, Cze. дахум, Lit. dešimtas, Мас. десеттиот, O.Ch.Sl. десатъ, Pol. dziesiąty, Rus. десятый, Ser. десети, Slo. deseto, Slv. desiaty, Ukr. десятий; C.I.L. III 13861, p 39; C.I.L. VIII 9377, p 39; C.I.L. III 3349, p 39; p 39; p 39; C.I.L. III, 8350, p 39; p 39; p 39; p 40; p 40; p 40; C.I.L. III 9024, p 40; p 40; p 40; p 41; p 41; C.I.L. V 893, p 44; V.T1.29.

35 *Denio, Dennata: day - Bel. дзень, Bos. dan, Bul. ден, Cro. dan, Cze. den, Lit. diena, Mac. ден, O.Ch.Sl. дьнь, Rus. день, Ser. дан, Slo. dan, Slv. deň, Ukr. день; C.I.L. III 2847, p 41; C.I.L. III 13278, p 41.

36 †Deuso, †Deusus: (1) robust - Bel. дужы, Rus. дюжий, Ukr. дужий; C.I.L. III 10883, p 42; C.I.L. III 5303, p 42; V.T1.28.

: (2) soul - Bel. душа, Bos. duša, Blg. душа, Cro. duša, Cze. duše, Lit. dvasia, Мас. душа, O.Ch.Sl. доуша, Pol. dusza, Rus. душа, Ser. душа, Slo. duša, Slv. duše, Ukr. душа; C.I.L. III 10883, p 42; C.I.L. III 5303, p 42; V.T1.28.

$37{ }^{\star}$ Dida, ${ }^{\star D}$ ita: child - Bel. дзіця, Bos. dijete, Bul. дете, Cro. dijete, Cze. dítě, Lit. duktè, Mac. дете, O.Ch.Sl. дъть, Pol. dziecko, Rus. дитя, Ser. дете, Slv. dieta, Ukr. дитя; C.I.L. V 1958, p 42; p 43.

38 Diteius, Ditica, Ditio, Dito, Ditueius, Ditus: children - Bel. дзеці, Bos. deca, Bul. деца, Cro. djeca, Cze. děti, Мac. деца, O.Ch.Sl. дъти, Pol. деца, Rus. дети, Ser. деца, Slv. deti, Ukr. діти; C.I.L. III 9032, p 43; C.I.L. V 461, p 43; C.I.L. V 1830, p 43; C.I.L. III 1927, p 44; C.I.L. III 10040, p 44; C.I.L. III D VI, p 44.

$39{ }^{\star}$ Domator Dommus, Dumma: home/house - Bel. дом, Bos. dom, Bul. дом, Cro. dom, Cze. dům, Мас. дом, O.Ch.Sl. домъ, Pol. dom, Rus. дом, Ser. дом, Slv. dom, Ukr. дім; C.I.L.V 449, p 44; C.I.L. V 443, p 44; C.I.L. III 2858, p 45.

$40 \dagger$ Drus(s)ius, Drus(s)ia: friends - Bel. друзья, Lit. draugai, Rus. друзья, Ukr. друзі; C.I.L. IX 505, p 44; C.I.L. IX 505, p 44.

41 †Ducetios: spirit - Bel. дух, Bos. duh, Bul. дух, Cro. duh, Cze. duch, Lit. dvasia, Mac. дух, O.Ch.Sl. доухъ, Pol. duch, Rus. дух, Ser. дух, Slo. duha, Slv. duch, Ukr. дух; p 45.

\section{E}

42 Egirus: urchin - Bos. jež, Lit. ežys, Rus. ёж, Ser. јеж, Slo. jež, Slv. ježko, Ukr. їжак; C.I.L. V 727, p 45 .

43 Elonia, †Flonia: fir - Bel. елка, Bos. jelka, Bul. ела, Cro. jela, Cze. jedle, Lit. egle, Mac. ела, Pol. jodła, Rus. ель, Ser. јела, Slo. jelka, Slv. jedla; p 46; C.I.L. V 2253, p 51.

44 Ettritus: nucleus - Bel. ядро, Bos. jezgra, Blg. ядро, Cro. jezgra, Cze. jádro, Mac. jадро, Pol. jądro, Rus. ядро, Ser. језгро, Slo. jedro, Slv. jadro, Ukr. ядро; p 49; V.T1.31.

45 Etuta: this - Bel. гэта, Cze. tento, O.Ch.Sl. тъ, Pol. to, Rus. этот, Slo. to, Slv. toto; p 49.

46 [Extionia]: to go - Bel. ехаць, Lit. eiti, O.Ch.Sl. ехати, Rus. ехать, Ukr. їхати; C.I.L. V 456, p

50. 


\section{Continued}

\section{F}

47 Fasaca, Fasena: face - Bel. фac, Rus. фac, Ukr. фac; C.I.L. V 410, p 50; C.I.L. III 12285, p 50.

48 Fata, Fato: veil - Bel. фата, Rus. фата, Ukr. фата; C.I.L. III 3134, p 50; C.I.L. III 1201434, p 50.

\section{G}

49 *Gillos: lived - Bel. жылі, Bul. живял, Cro. živjeli, Cze. žil, Pol. żył, Rus. жил, Slv. žíl; p 54.

50 *Glavus: head - Bel. галава, Bos. glava, Bul. глава, Cro. glava, Cze. hlava, Lit. galva, Mac.

главата, O.Ch.Sl. глава, Pol. głowa, Rus. голова, Ser. глава, Slo. glavo, Slv. hlava, Ukr. голова; p 55.

51 Grabon, Grabos, Grabovius: hornbeam - Bel. граб, Bos. graba, Bul. габър, Cro. grab, O.Ch.Sl. грабъ, Pol. grab, Rus. граб, Ser. граба, Slo. gaber, Slv. hrab, Ukr. граб; p 55; p 55; p 55.

$52 †$ Graecidius: buckwheat - Bel. грэчка, Lit. grikiai, Pol. gryka, Rus. греча, Ukr. гречка; C.I.L. IX 338, p 55.

53 Gresa: dream - Bel. грёза, O.Ch.Sl. гръза Rus. грёза, Ukr. греза; C.I.L. III 14538, p 55.

H

54 *Hanicus: to hunt - Bos. goniti, Blg. гоня, Cro. goniti, Cze. hon, Lit. guiti, O.Ch.Sl. гонити, Pol. uganiać, Rus. гнать, Ser. гонити, Slv. hon, Ukr. гнати; C.I.L. III 4367; p 56; V.T1.32.

55 Hoplon: stronghold - Bel. аплот, Rus. оплот, Ukr. оплот; p 56.

56 Hostila, Hostil(i)us, Hostius, Hostus: to remain/to stay - Bos. ostati, Blg. остават, Cro. ostati, Cze. zůstat, Мас. остане, O.Ch.S. стати, Rus. остаться, Ser. остати, Slo. ostati, Slv. ostat; C.I.L. III, 10746, p 56; C.I.L. V 4965, p 56; C.I.L. III 10726, p 56; C.I.L. V 431, p 56; V.T1.36.

$57 \dagger$ †tto, Ittu, †Iturius, Jettus: to go - Bel. ісці, Bos. ići, Blg. отида, Cro. ići, Cze. jít, Lit. eiti, Mac. Да оди, O.Ch.Sl. ити, Pol. iść, Rus. идти, Ser. иде, Slo. iti, Slv. íst', Ukr. йти; p 58; C.I.L. III, 4784, p 58; C.I.L. V 2036, p 59; C.I.L. III, 2768, p 58; V.T1. 39.

J

58 †Jadia: poison - Bel. яд, Cze. jed, Pol. jad, Rus. ядъ, Slv. jed, Ukr. яд'; C.I.L. III 2563, p 56.

59 *Jaemiota, †Jamusioi: hole - Bel. яма, Bos. jama, Bul. яма, Cro. jama, Cze. jáma, Mac. јама, O.Ch.Sl. Ама, Rus. яма, Ser. јама, Slo. jama, Slv. jama, Ukr. яма; C.I.L. III 6389, p 57; C.I.L. V 2780, p 57.

60 Jasus: clear - Bel. ясна, Bos. jasno, Bul. ясно, Cro. jasno, Cze. jasný, Mac. jacно, Pol. jasny, Rus. ясный, Ser. јасан, Slo. jasen, Slv. jasný, Ukr. ясний; C.I.L. III D LX, p 57.

61 Javia: reality - Bel. ява, Rus. явь, Ukr. ява; p 57.

62 Jutossica: stern - Rus. ют; C.I.L. 10174, p 59.

\section{K}

L

63 *Laeca: hunting dog - Bel. лайка, Rus. лайка, Ukr. лайка; C.I.L. V 1980, p 60.

$64{ }^{\star}$ Lannus: deer - Bel. алені, Bos. jelen, Blg. елен, Cro. jelen, Cze. jelen, Lit. elnias, Mac. елен, Pol. jeleń, Rus. олень, Ser. јелен, Slo. jelen, Slv. jeleň, Ukr. олень; CIL. V, 1. no. 3655, p 62; V.T1.43.

$65{ }^{\star}$ Lava, Lavincia, Lavius, Lavo, Laevicus, Laevonicus: lion - Bel. леў, Bos. lav, Blg. лъв, Cro. lav, Cze. lev, Lit. liūtas, Mac. лав, Pol. lew, Rus. лев, Ser. лав, Slo. lev, Slv. lev, Ukr. лев; C.I.L. XI 1626, p 64; C.I.L. III, 2773, p 64; C.I.L. III 1269, p 64; C.I.L. III 9846, p 64; C.I.L. V 449, p 60; C.I.L. XIV 263, p 61; V.T1.42.

66 Laid(i)us: boat - Bel. ладдзя, Bul. лодка, Cze. lod', O.Ch.Sl. ладии, Pol. łódka, Rus. ладья; C.I.L. III D VI, p 61. 


\section{Continued}

$67{ }^{\star}$ Lasagos, ${ }^{\star}$ Lasaiu, Lasimos, Lasinius, Laso, Lassonia: hole - Bel. лаз, Cze. laz, Pol. łaz, Rus. лаз, Ser. лаз, Slo. laz, Ukr. лаз; p 62; C.I.L. III 3817, p 63; p 63; C.I.L. 8856, p 63; C.I.L. III 3824, p 63; C.I.L. 10723, p 63.

68 [Lascontia]: weasel - Bel. ласкі, Bos. lasica, Cro. lasica, Cze. lasička, O.Ch.Sl. ласица, Pol. łasica, Rus. ласка, Ser. ласица, Slo. lasica, Slv. lasička, Ukr. ласка; C.I.L. III 3855, p 63.

69 Ledia, Ledietis, Ledrus: ice - Bel. лёд, Bos. led, Blg. лед, Cro. led, Cze. led, Lit. ledas, Мac. лед, Pol. lód, Rus. лёд, Ser. лед, Slo. led, Slv. lad, Ukr. лід; C.I.L. III. 4743, p 64; C.I.L. III 2778, p 64; C.I.L. III 9819, p 64; V.T1.44.

70 Levo, ${ }^{\star}$ Levonicus: lion - Bel. леў, Bos. lav, Blg. лъв, Cro. lav, Cze. lev, Lit. liūtas, Mac. лав, Pol. lew, Rus. лев, Ser. лав, Slo. lev, Slv. lev, Ukr. лев; C.I.L. V 61, p 66; C.I.L. XIV 1228, p 66; V.T1.42.

71 * Licaea, ${ }^{\star}$ Licaius, ${ }^{\star}$ Licaus, ${ }^{\star}$ Licca, ${ }^{\star}$ Liccaeus, Liccaius, ${ }^{\star}$ Licca(v) us, Licco, $\dagger$ Licovius, Licovia: (1) front - Bel. лік, Bos. lice, Bul. лице, Cro. lice, Mac. лице, O.Ch.Sl. лицє, Rus. лик, Ser. лице, Ukr. лик; C.I.L. V 8409, p 66; C.I.L. III 3224, p 66; p 66; p 67; C.I.L. III D C, p 66; C.I.L. V 1001, p 66; C.I.L. III 15101, p 67; C.I.L. III 3224, p 67; C.I.L. VIII 21041, p 67; C.I.L. III 5265, p 67; C.I.L. V 1958, p 67; V.T1.45.

: (2) personal - Bos. lični, Blg. личен, Cro. lični, Mac. лично, Rus. личный, Ser. лични; C.I.L. V 8409, p 66; C.I.L. III 3224, p 66; p 66; p 67; C.I.L. III D C, p 66; C.I.L. V 1001, p 66; C.I.L. III 15101, p 67; C.I.L. III 3224, p 67; C.I.L. VIII 21041, p 67; C.I.L. III 5265, p 67; C.I.L. V 1958, p 67; V.T1.45.

72 Lomoliavus: scrap - Bel. лом, Bul. лом Pol. złom, Rus. лом, Ser. лом, Slo. lòm, Ukr. лом; C.I.L. V 450, p 68.

73 Lonus: breast - Bel. ўлонне, Bul. лоно, Pol. łono, Rus. лоно, Ukr. лоно; C.I.L. III. C XX, p 68.

74 [Lubana], [Lubia], [Lubiamus]: (1) love - Bel. люб, Bos. ljubavi, Bul. любов, Cro. ljubav, Mac. убов, O.Ch.Sl. люб, Rus. люб, Ser. љубав, Slo. ljubezen, Ukr. люб; C.I.L. V 4637, p 69; C.I.L. V 5033, p 69; C.I.L. V 4992, p 69.

69; C.I.L. V 5033, p 69; C.I.L. V 4992, p 69

: (2) forehead - Bel. лоб, Rus. лоб, Ukr. лоб; C.I.L. V 4637, p

75 Lunnicus: moon - Bel. луна, Bul. луна, O.Ch.Sl. лоуна, Rus. луна, Slo. luna, Ukr. луна; C.I.L. III, 6412, p 69.

76 Lykkeios: (1) bow - Bel. лук, Bos. luk, Blg. лък, Cro. luk, Lit. lankas, Mac. лак, Pol. łuk, Rus. лук, Ser. лук, Slo. lok, Slv. lúk, Ukr. лук; р 69; V.T1.46.

: (2) onion - Bel. лук, Bos. luk, Bul. лук, Cro. luk, O.Ch.Sl. ınjən, Rus. лук, Ser. лук; p 69, V.T1.46.

\section{M}

$77{ }^{\star}$ Malabanus, Malennius, ${ }^{\star}$ Mollicius, ${ }^{\star}$ Mollo, ${ }^{\star}$ Mollonia: small - Bel. маленькі, Bos mali, Blg. малък, Cro. mali, Cze. malý, Mac. мал, O.Ch.Sl. малъ, Pol. mały, Rus. малый, Ser. мали, Slo. majhen, Slv. malý, Ukr. малий; C.I.L. V 150, p 71; p 71; C.I.L. V 587, p 76; C.I.L. V 1305, p 77; C.I.L. V 2974 p 77; C.I.L. V 3500, p77; V.T1.47.

78 Mandeta: vagina - Rus. манда, Ukr. манда; C.I.L. III $14617^{4}$, p 71.

$79{ }^{\star}$ Medaurus, Medella: (1) honey - Bel. мёд, Bos. med, Bul. мед, Cro. med, Cze. med, Lit. medus, Mac. мед, O.Ch.Sl. медъ, Pol. miód, Rus. мед, Ser. мед, Slo. med, Slv. med, Ukr. мед; C.I.L. VIII 2581, p 72; C.I.L. IX 390, p 72.

: (2) copper - Bel. медзь, Bul. мед, Cze. měd', Pol. miedź, Rus. медь, Slv. med, Ukr. мідь; C.I.L. VIII 2581, p 72; C.I.L. IX 390, p 72.

80 Melesocus, $†$ Mellito: chalk - Bel. мел, O.Ch.Sl. мълъкъ, Pol. miałki, Rus. мел, Ukr. мел; C.I.L. V 8127, p 73; C.I.L. III 2999; p 73.

81 *Metellus: blizzard - Bel. мяцеліца, Rus. метель, Ukr. заметіль; C.I.L. V 443, p 75. 


\section{Continued}

82 Milizza: dear - Bul. мил, Cze. milý, Lit. mielas, Mac. мил, Rus. милый, Slv. milý, Ukr. милий; C.I.L. III 8294, p 75.

83 Moicus, Moienus, Moilicus, Moiota: my - Bel. мой, Bos. moj, Bul. мой, Cro. moj, Cze. můj, Mac. мојата, O.Ch.Sl. мои, Pol. mój, Rus. мой, Ser. мој, Slo. moj, Slv. môj, Ukr. мій; C.I.L. III 2558, p 76; C.I.L. III 3647, p 76; C.I.L. V 587, p 76; C.I.L. III 3785, p 77.

84 Morcos: sea - Bel. мора, Bos. more, Blg. мope, Cro. more, Cze. moře, Mac. мope, Pol. morze, Rus. море, Ser. море, Slo. morje, Slv. more, Ukr. море; p 77; VT1.50.

85 [Mottu]: prodigal - Bel. мот, Bos. mot, Bul. мот, Cro. mot, Cze. mot, Lit. mot, Mac. мот, Pol. mot, Rus. мот, Ser. мот, Slo. mot, Slv. mot, Ukr. мот; C.I.L. III 5624, p 78.

$86 \dagger$ Mucatus: (1) flour - Bel. мука, Cze. mouka, O.Ch.Sl. мжка, Pol. mąka, Rus. мука, Ser. мука, Slo. moka, Slv. múka, Ukr. мука; p 78.

: (2) throe - Bul. мъки, Rus. мука, Slv. muky, p 78.

$87 \dagger$ †utronius: dreary - Mac. тмурна, Rus. муторный; C.I.L. IX 342, p 78.

88 Mutteia: dregs - Bel. муць, Pol. męty, Rus. муть, Ukr. мyть; C.I.L. V 2909, p 78.

$89 †$ Mytil(i)us: to wash - Bel. мыць, Cze. mytí, Mac. мие, O.Ch.Sl. мыти, Pol. umyć, Rus. мыть, Slv. umytie, Ukr. мити; p 78.

\section{N}

$90 \dagger$ Nammavos: to us - Bel. нам, Bos. nama, Cro. nama, Cze. nám, Rus. нам, Ser. нама, Slo. nam, Slv. nám, Ukr. нам; C.I.L. III 5901, p 79.

$91{ }^{\star}$ Naro: people - Bel. народ, Bul. народ, Cro. narod, O.Ch.Sl. народъ, Pol. naród, Rus. народ, Ukr. народ; p 79.

$92{ }^{\star}$ Nebres: careless - Bul. небрежен, Rus. небрежный; C.I.L. V 8133, p 79.

$93{ }^{\star}$ Nebus: sky - Bel. неба, Bos. nebo, Bul. небе, Cro. nebo, Cze. nebe, Mac. небо, O.Ch.Sl. небо, Pol. niebo, Rus. небо, Ser. небо, Slo. nebo, Slv. neba, Ukr. небо; C.I.L. III D LXXXIX, p 79.

94 Nevica, Nevilla, †Nevius, Nevola: new - Bel. новы, Bos. novo, Bul. нов, Cro. novi, Cze. nový, Lit. nauja, Мас. ново, О.Сh.Sl. новъ, Pol. nowy, Rus. новый, Ser. нови, Slo. novo, Slv. nový, Ukr. новий; C.I.L. V 453, p 79; C.I.L. III 3090, p 79; C.I.L. V 7641, p 79; C.I.L. V 498, p 80.

\section{O}

95 Oeplus, Oplica, Oplus, Oplusa, Op(p)alo: stronghold - Bel. аплот, Rus. оплот, Ukr. оплот; C.I.L. III 2891, p 80; C.I.L. III 3149, p 81; C.I.L. III, 3322, p 81; C.I.L. III, 3322, p 81; C.I.L. III 3866, p 82.

96 *Olcias: alder - Bos. jelša, Bul. елша, Cze. olše, Lit. alksnis, Pol. olcha, Rus. ольха, Ser. јелша, Slo. jelša, Slv. jelša, Ukr. ольха; p 80 .

97 [Ophelestes]: itinerant trader - Bel. офеня, Rus. офеня, Ukr. офеня; p 81.

98 Opia, Opiavus: experience - Bel. вопыт, Bul. опит, Rus. опыт; C.I.L. III 3144, p 81; C.I.L. III 10121, p 81; V.T1.56.

99 Oplica, Oplus, Oplusa, Op(p)alo: disgrace - Bel. апала, Rus. опала, Ukr.опала; C.I.L. III 3149, p 81; C.I.L. III 3322, p 81, C.I.L. III 3322, p 81, C.I.L. III 3866, p 82.

$100{ }^{*}$ Osthilos, Ostiala, Ostila, Ostilius, Ostus: to remain/to stay - Bos. ostati, Blg. остават, Cro. ostati, Cze. zůstat, Мас. остане, Rus. остаться, Ser. остати, Slo. ostati, Slv. Ostat;; p 83; C.I.L. V 2906, p 83; C.I.L. III 3853, p 83; C.I.L. V 2251, p 83; C.I.L. III 3806, p 83; V.T1.57.

101 Ovia, †Ovincius, †Ovinconius: sheep - Bel. авечка, Bos. ovce, Blg. овца, Cro. ovca, Cze. ovce, Lit. avys, Мас. овца, Pol. оwса, Rus. овца, Ser. овца, Slo. ovca, Slv. ovca, Ukr. овець; C.I.L. V 449, p 83; C.I.L. III 5139, p 83; C.I.L. VI 2613, p 83; V.T1.58. 


\section{Continued}

\section{$\mathbf{P}$}

$102{ }^{\star}$ Pacuvius: bye - Bel. пока, Rus. пока, Ukr. поки; p 83.

103 *Paeticus: the fifth - Bel. пяты, Bos. peti, Blg. петият, Cro. peti, Cze. pátý, Mac. петтиот, Рol. piąty, Rus. пятый, Ser. пети, Slo. peti, Slv. piaty, Ukr. п'ятий; C.I.L. V, 2035, p 83; V.T1.59.

104 Pai(i)o, Paius: (1) share - Bul. пай, Lit. pajus, Rus. пай, Ukr. пай; C.I.L. III 9839, p 84; C.I.L. V 1956, p 84.

: (2) obedient - Bel. пай, Rus. пай, Ukr. пай; C.I.L. III 9839, p 84; C.I.L. V 1956, p 84.

105 ^Palaus, ${ }^{\star}$ Palavellius: burnt - Rus. пал; C.I.L. XIV 1445, p 84; C.I.L. V 2392, p 84.

106 Panent-, Panentius, Panet- †Pantauchos, Pantia, Panto: antlers - Bel. панты, Rus. панты, Ukr. панти; C.I.L. III 2426, p 84; C.I.L. XI 93, p 84; p 85; p 85; C.I.L. III 9253, p 85; C.I.L. III 2786, p 85 .

107 ^Paravellius, ${ }^{\star}$ Paris: vapour - Bel. пара, Bos. para, Bul. пара, Cro. para, Cze. pára, Mac. пареа, O.Ch.Sl. паръ, Pol. para, Rus. пар, Ser. пара, Slo. pare, Slv. para, Ukr. пара; C.I.L. VI 2405, p $86 ; \mathrm{p} 86$.

108 Patalicus, Patalius, Patalus: marmalade - Rus. пат, Ukr. пат; C.I.L. V 452, p 86; C.I.L. III 13295, p 86; C.I.L. III 11661, p 87.

$109{ }^{\star}$ Phalacriōn, Phalacros, †Phalinos, [Phalios]: halyard - Bel. фал, Bul. фал, Pol. fał, Rus. фал, Ukr фал; p 88; p 88; p 89; p 89.

110 Platino, Plator, Platorius, Platura, Platurius, Platus, Platyr: pay - Bel. плата, Bos. plata, Bul. плащане, Cro. plata, Cze. plat, Mac. плата, Pol. zapłata, Rus. плата, Ser. плата, Slo. plača, Slv. výplata, Ukr. плата; C.I.L. III 2788, p 92; C.I.L. III 7821, p 92; C.I.L. III 2148, p 94; C.I.L. VI 24672, p 94; p 94; p 94; p 94.

111 Plaetor, Plaetorianus, Plaetorius, Pletor, Pletorius, Pletoronius: whip - Lit. plakti, Rus. плеть, Ukr. пліть; C.I.L. III 3149, p 91; p 91; C.I.L. III 2728, p 91; C.I.L. III 3804, p 95; C.I.L. VI 2544, p 95; C.I.L. V 455, p 95.

112 Plesont-: stretch of river - Bel. плёс, Rus. плёс, Ukr. плесо; C.I.L. III 3042, p 94.

113 †Potisus: sweat - Bel. пот, Bul. пот, Cze. pot, Мac. пот, O.Ch.Sl. потъ, Pol. pot, Rus. пот, Slo. pot, Slv. pot, Ukr. піт; C.I.L. III 2985, p 96.

114 Pravaus: right - Bel. правы, Bos. u pravu, Bul. правилно, Cro. pravo, Cze. pravá, Mac. право, O.Ch.Sl. правъ, Pol. prawo, Rus. правый, Ser. право, Slo. prav, Slv. správny, Ukr. правий; p 96.

115 Pyllos: saw - Bel. піла, Bos. pila, Cro. pila, Cze. pilka, Mac. пила, Pol. Piła, Rus. пила, Slv. Pílka, Ukr. пила; p 97.

\section{Q}

$\mathbf{R}$

$116{ }^{\star}$ Raecius, ${ }^{\star}$ Raecus, Recus: language - Bul. peч, Cze. řeč, O.Ch.Sl. ръчь, Rus. речь, Slv. reč; C.I.L. III, 3116, p 97; C.I.L. III 5789, p 97; C.I.L. III 9958, p 97.

117 †Ritius: to dig - Bel. рыць, Pol. ryć, Rus. рыть, Ukr. рити; C.I.L. V 1894, p 98.

$S$

118 Sallas, †Salynthios: salt - Bel. соль Bos. sol, Bul. сол, Cro. sol, Cze. sůl, Mac. сол, O.Ch.Sl. соль, Pol. sól, Rus. соль, Ser. co, Slo. sol, Slv. sol, Ukr. сіль; р 99; р 99; V.T1.71.

$119 †$ †amanna, †Samiarus: alone - Bos. sam, Blg. сам, Cro. sam, Cze. sám, Mac. caм, Pol. sam, Rus. сам, Ser. сам, Slo. sam, Slv. sám, Ukr. сам; C.I.L. III 2610, p 100; C.I.L. V, 1046, p 100; V.T1.66.

$120{ }^{*}$ Sanic(i)us: dignity - Bul. сан, Rus. сан, Ukr. сан; C.I.L. V 2433, p 100. 


\section{Continued}

$121 \dagger$ Sattava: hundred - Bel. сто, Bos. stotinu, Blg. сто, Cro. сто, O.ChSl.

съто, Pol. sto, Rus. сто, Ser. сто, Slo. sto, Slv. sto, Ukr. сто; C.I.L. V 3605, p 100; V.T1.67.

122 Sceno, Scenobarbus Scenobarvus, Scenua, Scenus: hay - Bel. сена, Bos. sijeno, Bul. сено, Cro. sijeno, Cze. seno, Lit. šienas, Мac. сено, O.Ch.Sl. съно, Pol. sienny, Rus. сено, Ser. сено, Slo. seno, Slv. seno, Ukr. сіно; C.I.L. V 186, p 101; C.I.L. III 1265, p 101; C.I.L. III 1265, p 101; C.I.L. XI 214, p 101; C.I.L. III 7372, p 101.

123 Scerdilaedus, Scerdis: heart - Bel. сэрца, Bos. srce, Bul. сърце, Cro. srce, Cze. srdce, Lit. širdis, Мac. срце, O.Ch.Sl. срьдьцє, Pol. serce, Rus. сердце, Ser. срце, Slo. srce, Slv. srdce, Ukr. серце; р 101; р 102.

124 *Scilus: awl - Bel. шыла, Bos. šilo, Bul. шило, Cro. šilo, Cze. šídlo, Mac. шило, O.Ch.Sl. шило, Pol. szydło, Rus. шило, Ser. шило, Slo. šilo, Slv. šidlo, Ukr. шило; C.I.L. III 4377, p 102.

$125{ }^{\star}$ Scirtios, ${ }^{\star S}$ cirtia, ${ }^{\star S}$ cirtos: breadth - Bul. широта, Cro. široka, Rus. широта, Ukr. широта; p 102; C.I.L. X 8264, p 102; p 102 .

$126{ }^{\star}$ Scordeia, ${ }^{\star}$ Scordias: soon - Bos. uskoro, Bul. скоро, Cro. uskoro, Mac. наскоро, Rus. скоро, Ser. ускоро, Slv. čoskoro, Ukr. скоро; p 103; p 103.

127 Sei(i)o: this - O.Ch.Sl. сь, Rus. сей, Ukr. сей; C.I.L. III 2756, p 103.

128 Selio: village - Bel. сяло, Bos. selo, Bul. село, Cro. selo, Mac. село, Rus. село, Ser. село, Ukr. село; C.I.L. III 8604, p 103.

129 †eminiacca: seven - Bel. сем, Rus. семь, Ukr. cim; C.I.L. IX 3486, p 103.

130 Sera, Serus: sulfur - Bel. cepa, Bul. сяра, Cze. síra, Lit. sieros, Pol. siarka, Rus. cepa, Slv. síra, Ukr. сірка; C.I.L. III 2787, p 103; p 103.

131 Sestus, Sexticus, Sexto, Sextus: sixth - Bel. шосты, Bos. šesto, Bul. шести, Cro. šesti, Cze. šestý, Lit. šeštas, Mac. шесто, O.Ch.Sl. шєстъ, Pol. szósty, Rus. шестой, Ser. шесто, Slo. šesti, Slv. šiesty, Ukr. шостий; C.I.L. III 9876, p 103; C.I.L. V 456, p 104; C.I.L. III 10079, p 104; C.I.L. III 2790, p 104.

132 †Sibalis: rapid - Bel. шыбко, Rus. шибкий, Ukr. шыбко; p 104.

133 † Silo: force - Bel. сіла, Bos. silu, Blg. сила, Cro. sila, Cze. síla, Mac. сила, Pol. siła, Rus. сила, Ser. сила, Slo. silo, Slv. sila, Ukr. сила; C.I.L. III 5165, p 104; V.T1.70.

134 *Sinus: son - Bel. сын, Bos. sin, Bul. син, Cro. sin, Cze. syn, Lit. sūnus, Mac. син, O.Ch.Sl. съінъ, Pol. syn, Rus. сын, Ser. син, Slo. sin, Slv. syn, Ukr. син; C.I.L. III 2778, p 105.

135 *Sipa, Sipo, †Sippas: thorn - Bel. шып, Bul. шип, Rus. шип, Ukr. шип; C.I.L. III 12014529, p 105; C.I.L. V 2327, p 105; p 105.

136 Sirras: cheese - Bel. сыр, Bos. sir, Bul. сирене, Cro. sir, Cze. sýr, Lit. sūris, Мас. сирење, O.Ch.Sl. сыръ, Pol. ser, Rus. сыр, Ser. сир, Slo. sir, Slv. syr, Ukr. сир; р 105.

137 Slator: gold - Bel. золата, Bos. zlato, Bul. злато, Cro. zlato, Cze. zlato, Mас. злато, O.Ch.Sl. злато, Pol. złoto, Rus. злато, Ser. злато, Slo. zlato, Slv. zlato, Ukr. золото; C.I.L. III 9810, p 105.

$138{ }^{*}$ Sola, †Solia: salt - Bel. соль, Bos. sol, Bul. сол, Cro. sol, Cze. Sůl, Mac. сол, O.Ch.Sl. соль, Pol. sól, Rus. соль, Ser. со, Slo. sol, Slv. sol', Ukr. сіль; C.I.L. III 787, p 106; C.I.L. III 5487, p 106; V.T1.71.

139 †ōnylos: dream - Bos. san, Bul. сън, Cro. san, Cze. sen, Mac. сон, O.Ch.Sl. сънъ, Pol. sen, Rus. сон, Ser. сан, Slo. sanje, Slv. sen, Ukr. сон; p 106.

140 *Stataria, Staticus, Statinius: become - Bel. стаць, Cze. stát se, O.Ch.Sl. стати, Рol. stają się, Rus. стать, Slv. stat’ sa, Ukr. стати; C.I.L. III 8323, p 106; C.I.L. III 2795, p 106; C.I.L. III 2790, p 106.

141 Stul-, *Stulio: chair - Bos. stolica, Bul. стол, Cro. stolica, Cze. stolice, Mac. стол, Rus. стул, Ser. столица, Slo. stol, Slv. stolica, Ukr. стілець; C.I.L. III 13205, p 106; C.I.L. V 2856, p 106.

$142 †$ Surco, ${ }^{\star}$ Suricus: marmot - Bel. сурок, Rus. сурок; p 107; C.I.L. III 4197, p 107. 


\section{Continued}

$143{ }^{\star}$ Surilla, ${ }^{\star}$ Surinus, ${ }^{\star}$ Surio, ${ }^{\star}$ Surus: severe - Bel. суровы, Blg. суров, Pol. surowy, Rus. суровый, Slv. surový, Ukr. суворий; C.I.L. III 4834, p 107; C.I.L. V 483, p 107; C.I.L. VI 3184, p 107; C.I.L. III 1189, p 108; V.T1.73.

144 Sutta, Suttihus, Suttis, Sutt(i)us: essence - Bos. suština, Cro. suština, Rus. суть, Ser. суштина, Ukr. суть; C.I.L. III 8021, p 109; C.I.L. III 4831, p 109; C.I.L. III 143167, p 107; p 107.

\section{$\mathrm{T}$}

$145{ }^{\star}$ Talasios, ${ }^{\star}$ Talasus, $†$ Tals(i)us: (1) hostage - Cro. talac, Rus. таль, Ser. талау, Slo. talca, Ukr. таль; p 110; C.I.L. V 2323, p 110; C.I.L. III 3811, p 110.

V 2323, p 110; C.I.L. III 3811, p 110.

: (2) thawed - Bel. талы, Rus. талый, Ukr. талий; p 110; C.I.L.

$146 \dagger$ Tata, Tato, Tatta, Tattaia, Tattaris, ${ }^{\star}$ Tattu, Tattuia, ${ }^{*}$ Tattus, $\dagger$ Tatusius: thief - Bel. тать, O.Ch.Sl. татъ, Rus. тать, Slo. tat, Ukr. тать; C.I.L. III 5504, p 111; C.I.L. 8095, p 111; C.I.L. III 12775, p 111; C.I.L. III 8342, p 111; C.I.L. III 12800, p 111; C.I.L. III 11600, p 111; p 111; C.I.L. III 4948, p 111; C.I.L. III 3191, p 111.

147 Telavia: body - Bel. цела, Bos. tijelo, Bul. тяло, Cro. tijelo, Cze. tělo, Mac. тело, O.Ch.Sl. тъло, Rus. тело, Ser. тело, Slo. telo, Slv. telo, Ukr. тіло; p 112.

148 Temans, Temeia, Temus: darkness - Bos. tama, Bul. тъмнина, Cro. tama, Cze. tma, Lit. tamsa, Мас. темнина, O.Ch.Sl. тьма, Rus. темь, Ser. тама, Slo. temo, Slv. tma, Ukr. темь; C.I.L. X 3666, p 112; p 112; C.I.L. III 8489, p 112.

149 Tergitio: (1) market - Bos. tržište, Bul. тържище, Cro. tržnica, Cze. tržiště, Lit. turgaviet, Pol. targ, Ser. тржиште, Slo. trg, Slv. trh; C.I.L. III 4251, p 112; V.T1.77.

: (2) bargain - Bel. торг, Cze. targ, Pol. targ, Rus. торг; C.I.L. III, 4251, p 112; V.T1.77.

$150{ }^{\star}$ Terna, ${ }^{\star}$ Ternila: blackthorn - Bel. цёрн, Bos. trn, Bul. трън, Cro. trn, Cze. trnka, Мас. трн, Pol. tarnina, Rus. тёрн, Ser. трњина, Slo. trn, Slv. trnka, Ukr. терен; C.I.L. V 4716, p 112; C.I.L. V 449, p 112.

$151 \dagger$ Tertaus, Tritanerus, Tritano, Tritanus, Triteuta, Tritos: third - Bel. трэці, Bos. treće, Bul. трето, Cro. treći, Cze třetí, Мас. Трети, O.Ch.Sl. трєти, Pol. trzeci, Rus. третий, Ser. трећи, Slo. tretji, Slv. tretí, Ukr. третій; C.I.L. XI 4092, p 112; C.I.L. III 2796, p 117; C.I.L. III 2792, p 117; p 117; p 117; C.I.L. III 6411, p 118; V.T1.76.

152 Testimos, Testo: father in law - Bel. цесць, Bos. tast, Cro. tast, Mac. test, Pol. test, Rus. тесть, Ser. тест, Slo. test, Slv. test, Ukr. тест; p 113; C.I.L. III 8326, p 113.

153 Trosantios, Trosius: rope - Bel. трос, Rus. трос, Ukr. трос; p 118; C.I.L. III 633 II 8, p 119.

154 Truppicus: corpse - Bel. труп, Bul. труп, Мас. труп, Pol. trup, Rus. труп, Slo. truplo, Ukr. труп; C.I.L. V 2435, p 119.

155 Tudania, Tudicius: there - Bel. туды, Rus. туда, Ukr. туди; C.I.L. III 2797, p 119; C.I.L. V 2515, p 119.

$156{ }^{\star}$ Turelius, Turia, Turica, Turo, Turoius, Turus: aurochs - Lit. tauras, O.Ch.Sl. туръ, Pol. tur, Rus. тyp; C.I.L. III 4150, p 120; C.I.L. III 2700, p 120; C.I.L. V 477, p 120; C.I.L. III 2794, p 120; C.I.L. III 10724, p 120; C.I.L. III 13295, p 120.

157 Tutia, ${ }^{\star}$ Tutor, ${ }^{*}$ Tutorina, ${ }^{\star}$ Tutorius: mulberry - Bos. dud, Cro. dud, Rus. тута, Ser. дуд, Ukr. тута; C.I.L. III. 10949, p121; C.I.L. IX 1920, p 121; C.I.L. IX 351, p 121; C.I.L. IX 24, p 121.

\section{U}

V

158 †Val(l)ius: wave - Bel. хваля, Bos. val, Bul. вълна, Cro. val, Cze. vlna, Rus. вал, Slo. val, Slv. vlna, Ukr. вал; C.I.L. III 6423, p 122.

$159 \dagger$ †anamiu: bath - Bel. банята, Blg. баня, Lit. vonia, Mac. бања, Rus. баня, Ukr. баня; C.I.L. III 4244, p 122; V.T1.79. 


\section{Continued}

160 Varieus, Varro: cook - Bel. вары, Cze. vařit, Lit. virëjas, Rus. вари, Slv. uvarit, Ukr. вари, p 123; C.I.L. III 8551, p 123.

$161 \nmid$ Veianius, †Veiedius, †Veienus, †Veionius, †Veius: blowing - Bel. веючы, Rus. вея, Ukr. вея; C.I.L. III 2600, p 123; C.I.L. V 1442, p 123; C.I.L. V 2724, p 123; C.I.L. V 3058, p 124; C.I.L. V 1356, p 124.

162 Venda, Vendes, Vendo: to lead - Bos. voditi, Blg. води, Cro. voditi, Cze. vedení, Lit. vadovauti, Мас. да води, Rus. водити, Ser. водити, Slo. voditi, Slv. vodit, Ukr. водити; C.I.L. V3425, p 124; C.I.L. III 13278, p 124; C.I.L. III 2796, p125; V.T1.82.

163 †Venesavos: crown - O.Ch.Sl. въньць, Pol. wieniec, Rus. венец, Ukr. вінець; p 125.

164 Verzo, ${ }^{*}$ Verzobius, Verzovia: top - Bel. верх, Bos. vrh, Bul. връх, Cro. vrh, Lit. viršuje, Pol. wierzch, Rus. верx, Ser. вpx, Slo. vrh, Ukr. вepx; C.I.L. III 9056, p 126; C.I.L. IX 2123, p 126; C.I.L. III 1217, p 126.

165 Vescleves: authoritative - Bel. важкі, Pol. ważki, Rus. веский; C.I.L. III 3038, p 126; V.T1.83.

$166 \dagger$ †eseca, ${ }^{\star}$ Veselia, ${ }^{\star}$ Vesidia, ${ }^{\star}$ Vesius: whole - Bel. весь, Lit. visas, Rus. весь, Ukr. весь; C.I.L. III 5922, p 127; C.I.L. III 3093, p 127; C.I.L. III 2525, p 127; C.I.L. III 1797, p 127.

167 Viniocus: wine - Bel. віно, Bos. vino, Blg. вино, Cro. vino, Cze. víno, Lit. vyno, Мас. вино, Pol. wino, Rus. вино, Ser. вино, Slo. vino, Slv. víno, Ukr. вино; C.I.L. III 3154, p 127; V.T1.84.

168 Virno, Virraus: vortex - Bel. вip, Bul. вихър, Cro. vir, Cze. vír, Lit. verpetas, O.Ch.Sl. верхъ, Pol. wir, Rus. вихрь, Ser. вир, Slv. vír, Ukr. вир; С.I.L. III 2897, p 127; C.I.L. V 3842a, p 127.

169 Volsetis, Volsimus, Volsius, Volso, Volsouna, Volsun-, Volsus: hair - Bel. валасоў, Cze. vlasy, O.Ch.Sl. власъ, Pol. włosy, Rus. волос, Slv. vlasy, Ukr. волось; C.I.L. III 3055, p 128; p 128; C.I.L. III 2617, p 128; C.I.L. III 2968a, p 128; C.I.L. III 3149, p 128; C.I.L. III 3151, p 128; C.I.L. III 2985, p 128.

170 Voltisa: power - Bos. vlast, Blg. власт, Cro. vlast, Pol. władza, Rus. власть, Ser. власт, Ukr. влада; C.I.L. III 2900, p 130; V.T1.85.

$171{ }^{*}$ Voranicca: crow - Bel. ворон, Bos. vrana, Bul. врана, Cro. vrana, Cze. vrána, Lit. varna, Мас. врана, O.Ch.Sl. врана, Pol. wrona, Rus. ворон, Ser. врана, Slo. vrana, Slv. vrana, Ukr. ворон; C.I.L. V 467, p 131.

172 Votticius, Votticia: behold - Rus. вот; C.I.L. V 1829, p 131; C.I.L. III 4735, p 131.

\section{W X Y}

Z

173 Zaca: sunset - Bel. закат, Rus. закат, Ukr. закат; C.I.L. III 12718, p 131.

174 Zaiio: rabbit - Bos. zec, Blg. заек, Cro. zec, Lit. zuikis, Mac. зајак, Pol., Rus. заяц, Ser. зец, Slo. zajec, Slv. zajac, Ukr заєць; C.I.L. III 2756, p. 131; V.T1.86.

175 Zanatis: to take - Bel. заняць, Rus. занять, Ukr. зайняти; C.I.L. III 14620, p 131.

176 Zorada: sunrise - Bel. зоръ, Bos. zora, Cro. zora, Lit. aušra, Mac. зора, Pol. zorza, Rus. зоръ, Ser. зора, Slo. zora, Ukr. зop'; C.I.L. III 14620, p 131.

Table 2. Language conventions.

\begin{tabular}{cccc}
\hline sure I. anthr. & * very prob. I. anthr. & † doubt. I. anthr. & [ ] to be seen as I. ant. \\
Bel. Belarusian & Bos. Bosnian & Blg. Bulgarian & Cro. Croatian \\
Cze. Czechosl. & Lit. Lithuanian & Mac. Macedonian & O.Ch.Sl. Old Church Slavonic \\
O.Blg. Old Bulg. & Pol. Polish & Rus. Russian & Ser. Serbian \\
Slo. Slovenian & Slv. Slovakian & Ukr. Ukrainian & \\
\hline
\end{tabular}




\section{Discussion}

The C.I.L. is a continuously updated Latin inscription catalogue covering the Roman Empire period and its provinces, therefore, it is not surprising that Table 1 I. anthroponyms are deeply latinised and graecized. The Slavic root for each I. anthroponym was identified by comparing it with corresponding lexemes in the present surviving Slavic languages (Table 2), this because ancient Slavic documents are rare (Ambrozic \& Tomezzoli, 2003) (Ambrozic, 2005) (Ambrozic et al. 2006) (Šavli et al., 1996) (Serafimov, 2006) (Serafimov, 2007a) (Serafimov, 2007b) (Serafimov \& Tomezzoli, 2009) (Serafimov \& Tomezzoli, 2012) (Tomažič \& Tomezzoli, 2003) (Tomezzoli, 2001) (Tomezzoli \& Čudinov, 2002) (Tomezzoli, Serafimov \& Vodopivec V, 2009) (Tomezzoli \& Serafimov 2013) (Vodopivec, 2008) (Vodopivec, 2009a) (Vodopivec, 2009b) and normally contain few lexemes, insufficient for meaningful comparisons with I. anthroponyms.

\subsection{Initial Considerations}

Similarly to what already observed in considering the V. anthroponyms (Stein \& Tomezzoli, 2020), the following is observed.

The concept of nature is present in: T1.1 hell T1.5 reality, T1.9 white, T1.10 birch, T1.11 spring, T1.14 pine, T1.15 shore, T1.20 mud, T1.21 stone, T1.23 carp, T1.25 hour, T1.27 root, T1.28 mountain, T1.35 day, T1.42 urchin, T1.43 fir, T1.44 nucleus, T1.51 hornbeam, T1.52 buckwheat, T1.59 hole, T1.60 clear, T1.61 reality, T1.64 deer, T1.65 lion, T1.67 hole, T1.68 weasel, T1.69 ice, T1.70 lion, T1.75 moon, T1.76(2) onion, T1.79(2) copper, T1.80 chalk, T1.81 blizzard, T1.84 sea, T1.93 sky, T1.101 sheep, T1.105 burnt, T1.107 vapour, T1.112 stretch of river, T1.118 salt, T1.122 hay, T1.130 sulfur, T1.135 thorn, T1.137 gold, T1.138 salt, T1.142 marmot, T1.148 darkness, T1.150 blackthorn, T1.156 aurochs, T1.157 mulberry, 158 wave, T1.164 top, T1.166 whole, T1.171 crow, T1.173 sunset, T1.174 rabbit, T1.176 sunrise.

The concept of person is present in: T1.3 quick, T1.6 without, T1.16 go away, $\mathrm{T} 1.33$ to oppress, T1.36(1) robust, T1.36(2) soul, T1.40 friends, T1.41 spirit, T1.46 to go, T1.47 face, T1.49 lived, T1.50 head, T1.53 dream, T1.56 to remain/to stay, T1.57 to go, T1.71(1) front, T1.71(2) personal, T1.73 breast, $\mathrm{T} 1.74(1)$ love, T1.74(2) forehead, T1.77 small, T1.78 vagina, T1.83 my, T1.86(2) throe, T1.91 people, T1.99 disgrace, T1.100 to remain/to stay, T1.102 bye, T1.113 sweat, T1.116 language, T1.119 alone, T1.120 dignity, T1.123 heart, T1.128 village, T1.133 force, T1.139 dream, T1.140 become, T1.145(1) hostage, T1.146 thief, T1.147 body, T1.154 corpse, T1.159 bath, T1.169 hair, T1.170 power.

The concept of personality is present in: T1.12 whim, T1.17 rowdy, T1.82 dear, T1.87 dreary, T1.92 careless, T1.98 experience, T1.104(2) obedient, T1.114 right, T1.143 severe, T1.165 authoritative.

The concept of family is present in: T1.7 dad, T1.34 the tenth, T1.37 child, T1.38 children, T1.39 home/house, T1.90 to us, T1.103 the fifth, T1.131 sixth, T1.151 third, T1.152 father in law.

The concept of profession is present in: T1.2 granary, T1.4 shout to dogs, T1.8 
trouble, T1. 13 fighter, T1.19 alcoolic drink, T1.22 shackles, T1.24 hut, T1.26 barn, T1.29 dot, T1.30 rank, T1.31 gift, T1.32 to give, T1.45 this, T1.48 veil, T1.54 to hunt, T1.55 stronghold, T1.58 poison, T1.62 stern, T1.63 hunting dog, T1.66 boat, T1.72 scrap, T1.76(1) bow, T1.79(1) honey, T1.86(1) flour, T1.88 dregs, T1.89 to wash, T1.94 new, T1.95 stronghold, T1.97 itinerant trader, T1.104(1) share, T1.108 marmalade, T1.109 halyard, T1.110 pay, T1.111 whip; T1.115 saw, T1.117 to dig, T1.121 hundred, T1.124 awl, T1.125 breadth, T1.126 soon, T1.127 this, T1.129 seven, T1.132 rapid, T1.136 cheese, T1.141 chair, T1.145(2) thawed, T1.149(1) market, T1.149(2) bargain, T1.153 rope, T1.155 there, $\mathrm{T} 1.160$ cook, $\mathrm{T} 1.161$ blowing, $\mathrm{T} 1.162$ to lead, $\mathrm{T} 1.163$ crown, $\mathrm{T} 1.167$ wine, $\mathrm{T} 1.168$ vortex, T1.172 behold, T1.175 to take.

\subsection{Intermediate Considerations}

Similarly to what already observed in considering the V. anthroponyms (Stein \& Tomezzoli, 2020), the following is observed.

T1.1 hell is linked to the ancient Gr. "AıInৎ and indicates the underworld, a concept passed lately to the Christian tradition as hell.

T1.3 quick is linked to the hydronyms Adriatic: the sea facing the ancient I. territories, to Adrias/Atrianus: a no longer existing, ancient channel of the today Po river delta, mentioned by Hecateus, Theopompus and Ptolemy (Wikipedia, 2018) and to Jantra a today's Blg. river, tributary of Danube, which was named Athrys by the ancient Thracians.

T1.71(1)/(2) front/personal, T1.84 sea, T1.142 marmot preserve the suffix -co present in today's Slavic personal anthroponyms like, for example: Vinko, Stanko and Slavko.

T1.7 dad, T1.11 spring, T1.15 shore, T1.30 rank, T1.31 gift, T1.47 face, T1.74(1)/ (2) love/forehead, T1.119 alone, T1.150 blackthorn, T1.157 mulberry, T1.169 hair preserve the suffix-na present in today's Slavic personal anthroponyms like, for exsmple: Dragana, Stana and Svetlana.

\subsection{Final Considerations}

In said publication (Krahe, 1929), the I. anthroponyms total is 899 which represents a good sample for a statistical consideration. The I. anthroponyms in Table 1 having Slavic roots are 413 which represent $45.93 \%$ of the I. anthroponyms in said publication. The $\mathrm{V}$. anthroponyms in Table 1 of the previous publication (Stein \& Tomezzoli, 2020), having Slavic roots is roughly $24 \%$ of the V. anthroponyms in the corresponding publication (Pauli, 1891). This permits to estimate that during the Roman Empire period, i.e. about five cen., roughly $46 \%$ of the I. population and $24 \%$ of the V. population had Slavic ascendancies. This permits therefore to reply to the above question (Pigozzo, 2020) that ancient I. and V. were related through their Slavic ascendancies.

\section{Conclusion}

Said $46 \%$ of I. population and said $24 \%$ of V. population having Slavic ascendan- 
cies, during the Roman Empire period, highlight that Slavs were already present in areas incorporated by the Roman Empire well before the VII cen. A.D., the date, according to the generally accepted theory, of the Slav late arrival in Europe. The logical consequence is that this theory is wrong and should be rejected.

\section{Conflicts of Interest}

The authors declare no conflict of interests regarding the publication of this paper.

\section{References}

Ambrozic, A. (2005). The "Warrior" Stele from Lemnos. In Origin of the Slovenes. Proceedings of the Third International Topical Conference Ancient Settler of Europe (pp. 107-120). Ljubljana: Založništvo Jutro.

Ambrozic, A., \& Tomezzoli, G. (2003). The "Tavola da Este" inscription. In Origin of the Slovenes. Proceedings of the International Workshop Traces of European Past (pp. 132-146). Ljubljana: Založništvo Jutro.

Ambrozic, A., Serafimov, P., \& Tomezzoli, G. (2006). The Venetic Inscription ES 120 on the Cup of "Scolo di Lozzo". In Origin of the Slovenes. Proceedings of the Fourth International Topical Conference Ancient Inhabitants of Europe (pp. 166-173). Ljubljana: Založništvo Jutro.

Krahe, H. (1929). Lexikon Altillyrischer Personennamen. Indogermanische Bibliothek. Heidelberg: Carl Winter's Universitätbuchhandlung.

Pauli, C. (1891). Die Veneter und Ihre Schrftdenkmäler. Altitalische Forschungen. Leipzig: Johan Ambrosius Barth.

Pigozzo Bernardi, G. (2020). Alle origini della civiltà veneta. Dalla lingua antica ai dialetti veneto-friulani. Piazza Editore.

Šavli, J., Bor, M., \& Tomažič, I. (1996). Veneti. First Builders of European Community. Wien: Editiones Veneti.

Serafimov, P. (2006). The Sitovo Inscription. In Origin of the Slovenes. Proceedings of the Fourth International Topical Conference Ancient Inhabitants of Europe (pp. 198-204). Ljubljana: Založništvo Jutro.

Serafimov, P. (2007a). Steinberg Inscription. In Origin of the Slovenes. Proceedings of the Fourth International Topical Conference Ancient Inhabitants of Europe (pp. 174-182). Ljubljana: Založništvo Jutro.

Serafimov, P. (2007b). Translation of the Eteocretan EPIOI Inscription. In Origin of the Slovenes. Proceedings of the Fifth International Topical Conference Origin of Europeans (pp. 176-183). Ljubljana: Založništvo Jutro.

Serafimov, P., \& Tomezzoli, G. (2009). The Inscription from Tell El-Dab'a. In Origin of the Slovenes. Proceedings of the Seventh International Topical Conference Origin of Europeans (pp. 89-96). Ljubljana: Založništvo Jutro.

Serafimov, P., \& Tomezzoli, G. (2012). New Reading of the Linear a Inscription on the Golden Pin CR-ZF-1 from Crete. In Origin of the Slovenes. Proceedings of the Tenth International Topical Conference Origin of Europeans (pp. 83-90). Ljubljana: Založništvo Jutro.

Stein, R. S., \& Tomezzoli, G. T. (2020). Venetic Personal Anthroponyms. Archaeological Discovery, 8, 135-146. https://doi.org/10.4236/ad.2020.82008

Tomažič, I., \& Tomezzoli, G. (2003). The Inscription Pauli No. 39. In Origin of the Slo- 
venes. Proceedings of the International workshop Traces of European Past (pp. 147157). Ljubljana: Založništvo Jutro.

Tomezzoli, G. (2001). About Two Magre-Rhaetic Inscriptions in the Civic Natural History Museum in Verona. In Origin of the Slovenes. Proceedings of the First International Topical Conference the Veneti within the Ethnogenesys of the Central-European Population (pp. 182-187). Ljubljana: Založništvo Jutro.

Tomezzoli, G., \& Čudinov, V. A. (2002). The "Spada di Verona". In Origin of the Slovenes. Proceedings of the Conference Ancient Settlers of Central Europe (pp. 65-73). Ljubljana: Založništvo Jutro.

Tomezzoli, G., \& Serafimov, P. (2013). The Linear-A Inscriptions II.1, II.2 from the Palace of Knossos. In Origin of the Slovenes. Proceedings of the Eleventh International Topical Conference Origin of Europeans (pp. 105-114). Ljubljana: Založništvo Jutro.

Tomezzoli, G., Serafimov, P., \& Vodopivec, V. (2009). Two Noricum Inscriptions. In Origin of the Slovenes. Proceedings of the Seventh International Topical Conference Origin of Europeans (pp. 119-127). Ljubljana: Založništvo Jutro.

Vodopivec, V. (2008). Retijski Napis Pustertal. In Origin of the Slovenes. Proceedings of the Sixth International Topical Conference Origin of Europeans (pp. 137-141). Ljubljana: Založništvo Jutro.

Vodopivec, V. (2009a). Napisi na Ptujskih Olienkah. In Origin of the Slovenes. Proceedings of the Seventh International Topical Conference Origin of Europeans (pp. 141-147). Ljubljana: Založništvo Jutro.

Vodopivec, V. (2009b). Najdaljši Venetski Napis. In Origin of the Slovenes. Proceedings of the Seventh International Topical Conference Origin of Europeans (pp. 97-118). Ljubljana: Založništvo Jutro.

Wikipedia (2018). Adria (River). https://en.wikipedia.org/wiki/Adria (river)

Wilkes, J. (1992). The Illyrians. The Peoples of Europe. Oxford \& Cambridge, MA: Blackwell. 